\section{UCDNN}

LIBRARY
University of Connecticut OpenCommons@UConn

Department of Natural Resources and the

July 2005

\title{
Position Errors Caused by GPS Height of Instrument Blunders
}

Thomas H. Meyer

University of Connecticut, thomas.meyer@uconn.edu

April Hiscox

University of Connecticut

Follow this and additional works at: https://opencommons.uconn.edu/thmeyer_articles

\section{Recommended Citation}

Meyer, Thomas H. and Hiscox, April, "Position Errors Caused by GPS Height of Instrument Blunders" (2005). Thomas H. Meyer's Peer-reviewed Articles. 4.

https://opencommons.uconn.edu/thmeyer_articles/4 


\section{SURVEY REVIEW}

No.298

Vol.38

\section{CONTENTS (part)}

Position errors caused by GPS height of instrument blunders

262

T H Meyer and A L Hiscox

This paper was published in the October 2005 issue of the UK journal, SURVEY REVIEW. This document is the copyright of CASLE. Requests to make copies should be made to the Editor, see www.surveyreview.org for contact details.

The Commonwealth Association of Surveying and Land Economy does not necessarily endorse any opinions or recommendations made in an article, review, or extract contained in this Review nor do they necessarily represent CASLE policy

(C) CASLE 2005 


\title{
POSITION ERRORS CAUSED BY GPS HEIGHT OF INSTRUMENT BLUNDERS
}

\author{
T. H. Meyer and A. L. Hiscox \\ Department of Natural Resources Management and Engineering \\ University of Connecticut
}

\begin{abstract}
Height of instrument (HI) blunders in GPS measurements cause position errors. These errors can be pure vertical, pure horizontal, or a mixture of both. There are different error regimes depending on whether both the base and the rover both have HI blunders, if just the base has an HI blunder, or just the rover has an HI blunder. The resulting errors are on the order of $30 \mathrm{~cm}$ for receiver separations of $1000 \mathrm{~km}$ for an HI blunder of $2 \mathrm{~m}$. Given the complicated nature of the errors, we believe it would be difficult, if not impossible, to detect such errors by visual inspection. This serves to underline the necessity to enter GPS HIs correctly.
\end{abstract}

\section{INTRODUCTION}

In surveying situations where a "flat Earth" assumption is appropriate, it is wellknown that the height of instrument (HI) is essentially irrelevant for computing horizontal coordinates when using opto-mechanical instruments such as total stations [8]. Although completely rigorous treatments of trigonometric levelling are wellknown (e.g., see [4], pp.173-176), such treatments require knowledge not typically available in the field to a practicing surveyor, such as the geodesic distance between stations, geodetic latitude at both stations, deflection of the vertical at the observing station, the ellipsoid height of the observing station, and the radius of curvature in the azimuth at both stations. In practice, a total station makes a flat-Earth assumption so that the vertical components of measurements can be removed with simple trigonometry. This practice essentially projects the measurements into the horizontal plane defined by the local gravitational normal vector. Therefore, the height of the instrument is irrelevant for computing horizontal coordinates under a flat-Earth assumption. However, as far as is known to the authors, it has not been documented that the height of a GPS antenna (i.e. the vertical distance from the marker to the phase centre of a GPS antenna) effects the horizontal coordinates derived through GPS surveying and that ignoring GPS HI introduces horizontal position errors.

\section{BACKGROUND}

It may seem paradoxical at first that ignoring the GPS phase centre height would cause a horizontal position miscalculation. After all, if we assume that the GPS antenna range pole was levelled properly, the blunder is purely in the vertical and ought not to effect horizontal positions. The fallacy in this line of thinking stems from ignoring the fact that GPS positions are always inherently three-dimensional [8]. GPS positions are derived using satellites as moving monuments, whose instantaneous position is calculated either from the broadcast ephemerides or with post-processed precise ephemerides (e.g., see [9]). These positions are realized in a geocentric Cartesian coordinate system (XYZ) based on the WGS 84 datum [5], [7]. Although $\mathrm{XYZ}$ coordinates can easily be converted to a local north-east-up framework by simply rotating the coordinate system (e.g., see [13]), the coordinates remain inherently three- 
dimensional and changes in the local geodetic normal coordinate (i.e., up) can effect the other two. The problem is essentially the same as the skew of the normals [12], p. 141). The error caused by the skew of the normals depends on baseline length and, for short baselines, can often safely be ignored. However, unlike the situation with optomechanical surveying instruments whose baseline length is limited by inter-station visibility, GPS can be used to measure baselines thousands of kilometres in length. With such long baselines, the skew of the normals cannot be ignored and its effect amplifies GPS HI blunders, as will be shown.

The position being measured by a GPS receiver is located at the phase centre of the GPS antenna, being that point in space at which the antenna detects the radio signal broadcast from the satellites. The location of the phase centre differs from antenna to antenna, but it is located close to the antenna's conducting surface. The distance and direction of the phase centre to the mechanical centre of the antenna is established by calibration. The distance from the mechanical centre of the antenna to the antenna's reference point (ARP) is determined by the antenna's mechanical design. The distance from the ARP to the survey marker is related to the size of the device holding the antenna (e.g., a slant distance to the edge of the tripod face or to the antenna's ground plane or the length of a range pole). Thus, the GPS HI is the sum of these distances and will hereafter simply be referred to as GPS HI.

The effect of GPS antenna heights for computing coordinates is considered in standard works, such as [10]. The equations presented in Strang and Kai are given in the XYZ coordinate system and are compact and elegant. However, because we are interested in drawing a distinction between horizontal and vertical errors, we will work with geodetic coordinates. As will be shown in the next section, we adopt a more elaborate notation to highlight the exact places where the problems occur. Interestingly, in [10], the authors have implicitly made a "flat-Earth" assumption by using the same surface normal vector for both receivers. Therefore, using their equations creates exactly the sort of problem detailed in this paper (the authors indicated that they will update their equations in the next edition of their book [11]).

\section{HOW COORDINATES ARE COMPUTED}

The task of computing position coordinates from GPS measurements can be conceptualized as converting positions between geodetic, local horizontal geodetic, and geocentric Cartesian coordinate systems. To enumerate these conversions we adopt the following notation. Points and vectors are denoted by lower case bold face, e.g., p. Superscripts denote coordinate systems, e.g., $\mathbf{p}^{x y z}$. We denote a coordinate transformation operator by $\chi$, e.g., $\mathbf{p}^{\text {to }}=\chi^{\text {from } \rightarrow{ }^{t o}}\left(\mathbf{p}^{\text {from }}\right)$ denotes a mapping of point $\mathbf{p}$ from coordinate system from to coordinate system to. We begin by reviewing the relevant formulae.

Let $\varphi, \lambda, h$ denote geodetic latitude, longitude, and ellipsoidal height, respectively. Then, geodetic coordinates are converted to geocentric Cartesian coordinates using Helmert's projection [3]. For $\mathbf{p}^{\varphi \lambda \mathrm{h}}=(\varphi, \lambda, h)$,

$$
\begin{aligned}
& x=(\eta+h) \cos \phi \cos \lambda \\
& y=(\eta+h) \cos \phi \sin \lambda \\
& z=\left(\left(1-e^{2}\right) \eta+h\right) \sin \phi
\end{aligned}
$$

where $x, y, z$ are geodetic Cartesian coordinates, $e^{2}$ is the (first) eccentricity of the ellipsoid squared, and $\eta$ is the radius of curvature in the prime vertical at $\varphi$. Denote these formulae as $\mathbf{p}^{x y z}=\chi^{\varphi \lambda h \rightarrow x y z}\left(\mathbf{p}^{\varphi \lambda h}\right)$. 
The inverse problem of converting geocentric Cartesian coordinates to geodetic coordinates has no direct solution [4]. Although a great amount of research has been directed at this problem, [1] developed an iterative approach that is accurate to $1 \mu \mathrm{m}$ without iteration. Bowring's method is so widespread that it might be considered an industry standard [6] so we will use it in this work. Let $\rho=\sqrt{x^{2}+y^{2}}$. Then, for $\mathbf{p}^{x y z}=$ $(x, y, z)$, Bowring's method is

$$
\begin{aligned}
& \lambda=\arctan (y / x) \\
& \beta=\arctan \left(\frac{z}{(1-f) \rho}\right) \\
& \phi=\arctan \left(\frac{z+\varepsilon^{2} b \sin ^{3} \beta}{\rho-a e^{2} b \cos ^{3} \beta}\right) \\
& h=\frac{\rho}{\cos \phi}-v
\end{aligned}
$$

where $a$ and $b$ denote the lengths of the semi-major and semi-minor axes of the reference ellipsoid, respectively, $f=(a-b) / a$ is the flattening, and $\varepsilon=e^{2} /\left(1-e^{2}\right)$ is the second eccentricity of the ellipsoid. Denote these formulae as $\mathbf{p}^{\varphi \lambda \mathrm{h}}=\chi^{x y z \rightarrow \varphi \lambda \mathrm{h}}\left(\mathbf{p}^{x y z}\right)$.

It will be necessary to convert $X Y Z$ geocentric coordinates to a local horizontal geodetic system ( $n$-axis positive towards north, $e$-axis positive towards east, and $u$-axis positive up along the ellipsoid normal). This is accomplished by applying the following formulae, which show that the $N E U$ local horizontal coordinate system is merely the $X Y Z$ coordinate system translated and rotated to a new position and orientation. Importantly, its shape is not distorted. Following [13], if $\mathbf{o}=\left(x_{0}, y_{0}, z_{0}\right)=$ $\left(\varphi_{0}, \lambda_{0}\right)$ are the geocentric and geodetic coordinates of the origin of the local $N E U$ coordinate system and $\mathbf{p}^{x y z}=(x, y, z)$ are the geocentric coordinates of the point of interest, then

$$
\left[\begin{array}{l}
e \\
n \\
u
\end{array}\right]=\left[\begin{array}{ccc}
-\sin \lambda_{0} & \cos \lambda_{0} & 0 \\
-\sin \phi_{0} \cos \lambda_{0} & -\sin \phi_{0} \sin \lambda_{0} & \cos \phi_{0} \\
\cos \phi_{0} \cos \lambda_{0} & \cos \phi_{0} \sin \lambda_{0} & \sin \phi_{0}
\end{array}\right]\left[\begin{array}{l}
x-x_{0} \\
y-y_{0} \\
z-z_{0}
\end{array}\right]=\mathbf{M}\left[\begin{array}{l}
x-x_{0} \\
y-y_{0} \\
z-z_{0}
\end{array}\right]
$$

where $(e, n, u)$ are the local geodetic coordinates of $(x, y, z)$. Denote this equation as $\mathbf{p}^{\text {neu }}=\chi_{\mathbf{0}}^{x y z \rightarrow \text { пеи }}\left(\mathbf{p}^{x y z}\right)$.

Conversion from $N E U$ to $X Y Z$ is accomplished with the inverse of equation (2), being

$$
\left[\begin{array}{l}
x \\
y \\
z
\end{array}\right]=\mathbf{M}^{-1}\left[\begin{array}{l}
e \\
n \\
u
\end{array}\right]+\left[\begin{array}{l}
x_{0} \\
y_{0} \\
z_{0}
\end{array}\right]
$$

Denote this equation as $\mathbf{p}^{x y z}=\chi_{\mathbf{0}}^{\text {neu } \rightarrow x y z}\left(\mathbf{p}^{\text {neu }}\right)$.

We now consider the problem of computing coordinates using GPS observables. Our formulae assume that the deflection of the vertical is zero in order to focus on the principle issue, which is the effect of HI blunders. Strictly speaking, one ought to use the vectors defined by the deflection of the vertical at the base and the rover instead of the ellipsoidal surface normal vectors. However, the difference between the two amounts to a horizontal difference of $0.3 \mathrm{~mm}$ for a 2-metre range pole under a deflection of 30". Therefore, we ignore this difference to focus on HI blunders. Suppose there are two GPS receivers whose antennas are affixed atop two range poles that have been levelled properly over two survey markers, $\mathbf{a}$ and $\mathbf{b}$. Let $\mathbf{a}_{\phi}^{x y z}$ and $\mathbf{b}_{\phi}^{x y z}$ 
denote the $X Y Z$ coordinates of the phase centres of the antennas set up over $\mathbf{a}$ and $\mathbf{b}$, respectively. The single / double / triple differencing solution of the observations collected by these receivers results in an $X Y Z$ vector, $\mathbf{v}=\mathbf{b}_{\phi}^{x y z}-\mathbf{a}_{\phi}^{x y z}$, being the separation of the two phase centres, not their location in space. Without loss of generality, assume the geodetic position of survey marker $\mathbf{a}$ is known. Then b's geodetic coordinates can be found by the following procedure. Let $H I_{\mathbf{a}}$ and $H I_{\mathbf{b}}$ denote the height of the instruments over $\mathbf{a}$ and $\mathbf{b}$, respectively.

1. Consider a $N E U$ coordinate system whose origin is at a. Obviously, a's coordinates in such a system are $(0,0,0)$. The coordinates of $\mathbf{a}_{\phi}^{\text {neu }}$ are exactly $\left(0,0, H I_{\mathbf{a}}\right)$. The $X Y Z$ coordinates of $\mathbf{a}_{\phi}^{\text {neu }}$ are found by applying equation (3) to $\left(0,0, H I_{\mathbf{a}}\right)$ using $\mathbf{a}$ as the local origin.

2. Add $\mathbf{v}$ to $\mathbf{a}_{\phi}^{x y z}$ to produce $\mathbf{b}_{\phi}^{x y z}$, the $X Y Z$ coordinates of the phase centre of the antenna setup over $\mathbf{b}$.

3. Transform $\mathbf{b}_{\phi}^{x y z}$ to a $N E U$ coordinate system whose origin is at $\mathbf{b}$ by applying equation (2). Denote these coordinates by $\mathbf{b}_{\phi}^{\text {neu }}$.

4. Subtract $H I_{\mathbf{b}}$ from the up coordinate of $\mathbf{b}_{\phi}^{\text {neu }}$. This results in the $N E U$ coordinates of b, $\mathbf{b}^{\text {neu }}$.

5. Transform $\mathbf{b}^{\text {neu }}$ to geocentric $X Y Z$ (3) and thence to geodetic (1).

This algorithm can be expressed as

$$
\mathbf{b}^{\phi \lambda h}=\chi^{x y z \rightarrow \phi \lambda h}\left(\chi_{\mathbf{b}}^{\text {neu } \rightarrow x y z}\left[\chi_{\mathbf{b}}^{x y z \rightarrow n e u}\left\{\chi_{\mathbf{a}}^{\text {neu } \rightarrow x y z}\left(\left(0,0, H I_{\mathbf{a}}\right)\right)+\mathbf{v}\right\}-\left(0,0, H I_{\mathbf{b}}\right)\right]\right)
$$

$\mathrm{HI}$ blunders are created by using incorrect values for $H I_{\mathbf{a}}, H I_{\mathbf{b}}$, or both.

The effect of an HI blunder is to place $\mathbf{v}$ at the wrong location. For example, if the HI of both antennas is mistakenly taken to be zero, then $\mathbf{v}$ conceptually will be placed on the markers instead of at the phase centres. At first blush this might not seem to be a problem. After all, what has happened is that $\mathbf{v}$ has been translated down from the phase centres to the markers, which would seem to be the desired effect anyway. Indeed, if the earth had no curvature, then there would be no problem. However, the curvature of the Earth ensures that the up direction at $\mathbf{a}$ and $\mathbf{b}$ are different so this simple translation does not capture the correct geometry.

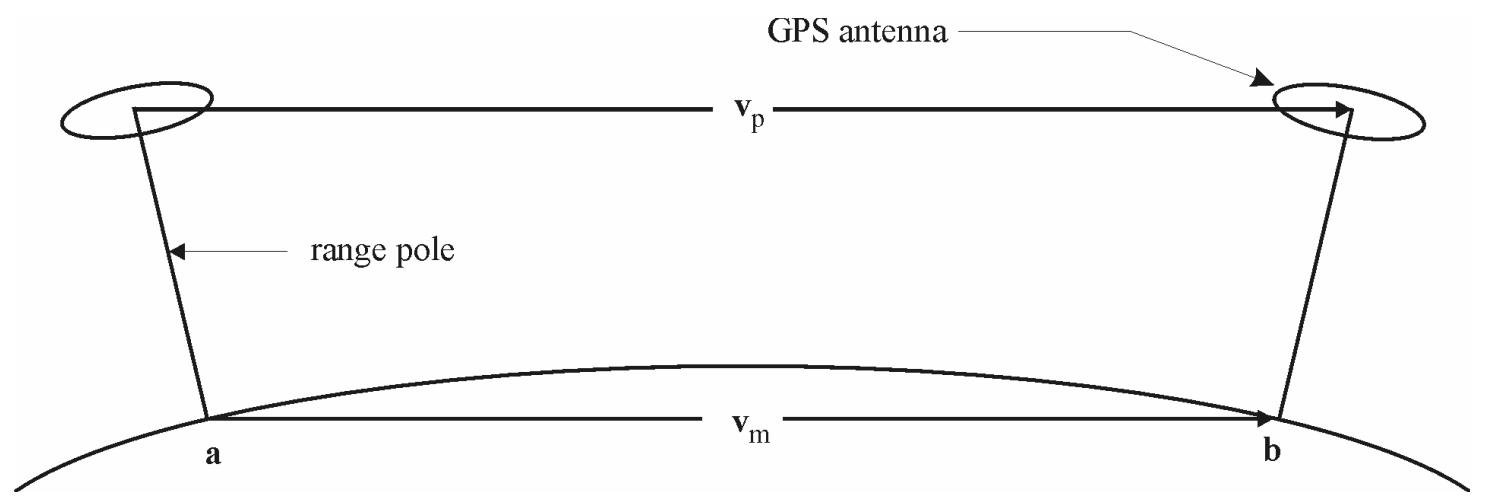

Fig. 1: The vector between the phase centres of two GPS antennas $\left(\mathbf{v}_{\mathrm{p}}\right)$ and the corresponding reduced vector between the markers $\left(\mathbf{v}_{\mathrm{m}}\right)$.

The phase centres of the antennas are farther apart in the $\mathrm{XYZ}$ coordinate system than are their respective markers. It is necessary to reduce the distance between the phase centres down to the markers, not merely translate the difference vector down. The 
situation is illustrated in Figure 1. In this Figure, $\mathbf{v}_{\mathbf{p}}$ is the vector between the phase centres and $\mathbf{v}_{\mathbf{m}}$ is the reduced vector between the markers. Merely adding $\mathbf{v}$ to $\mathbf{a}$ shifts the horizontal coordinates of $\mathbf{b}$ to $\mathbf{c}$ as shown in Figure 2.

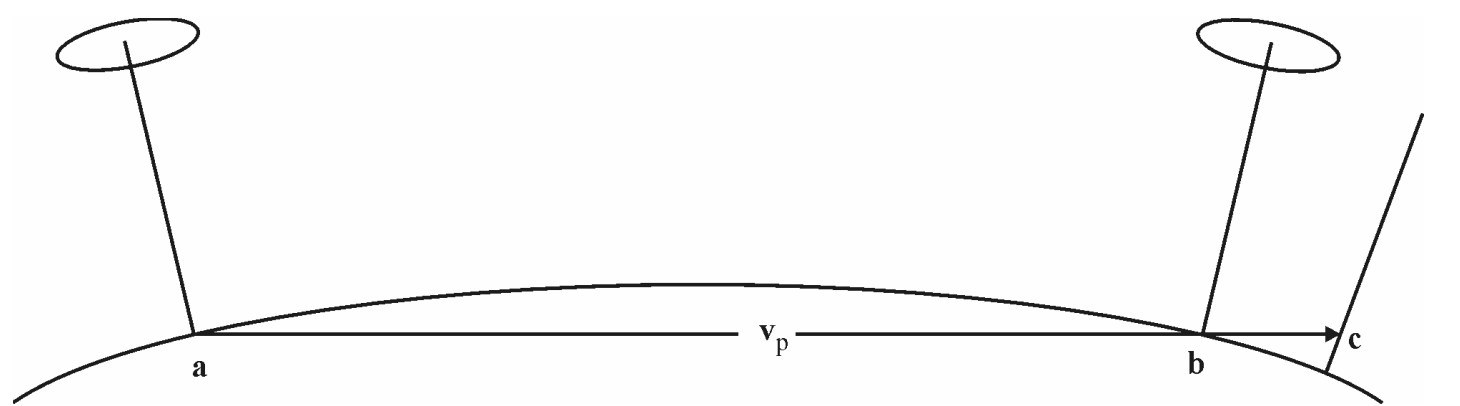

Fig. 2: Adding $\mathbf{v}_{\mathrm{p}}$ to $\mathbf{a}$ is not a reduction and does not result in the correct location of $\mathbf{b}$. Instead, it results in an offset position $\mathbf{c}$. Note that c's position is incorrect both horizontally and vertically.

\section{HI BLUNDER ANALYSES}

The HI of a receiver can be reported too high, too low, or correctly. Therefore, for a pair of receivers, there are seven possible blunder combinations (a total of $2^{3}=8$ combinations minus the correct/correct combination). It happens that there are three distinct error regimes for these combinations depending on the kind of error that occurred at the receiver that is used as the origin of $\mathbf{v}$. Each regime will be considered in turn.

Although there is not necessarily any clear notion of a "base" and "rover" receiver distinction with GPS surveying, we will adopt that nomenclature to mean the following. When the GPS post-processing software computes $\mathbf{v}$, it must do so with one phase centre at $\mathbf{v}$ 's origin and the other at $\mathbf{v}$ 's head. We will call the receiver at $\mathbf{v}$ 's origin the "base" receiver and the receiver at v's head the "rover." This language is useful because the three error regimes depend upon the nature of the error at the base receiver.

\section{No error at the base}

If there is an HI blunder only at the rover, then we can modify equation (4) by adding a blunder constant to the true $\mathrm{HI}$ at station $\mathbf{b}$ resulting in $\mathbf{b}_{c}^{\phi \lambda h}=\chi^{x y z \rightarrow \phi \lambda h}\left(\chi_{\mathbf{b}}^{n e u \rightarrow x y z}\left[\chi_{\mathbf{b}}^{x y z \rightarrow n e u}\left\{\chi_{\mathbf{a}}^{\text {neu } \rightarrow x y z}\left(\left(0,0, H I_{\mathbf{a}}\right)\right)+\mathbf{v}\right\}-\left(0,0, H I_{\mathbf{b}}+c_{\mathbf{b}}\right)\right]\right)$

where $c_{\mathbf{b}}$ is a real constant, and $\mathbf{b}_{c}^{\phi \lambda h}$ denotes the incorrectly computed position of $\mathbf{b}$; the subscript $c$ indicates that the base $\mathrm{HI}$ is correct. When $c_{\mathbf{b}}>0$, then the reported HI is too high and when $c_{\mathbf{b}}<0$, then the reported $\mathrm{HI}$ is too low. See Figure 3.

Examination of equation (4a) immediately reveals that $\mathbf{b}_{c}^{\phi \lambda h}$ differs from $\mathbf{b}^{\phi \lambda h}$ only in the normal direction. This is true because the head of $\mathbf{v}$ has been placed correctly at $\mathbf{b}_{\phi}^{\phi \lambda h}$. The subsequent subtraction of $\left(0,0, H I_{\mathbf{b}}\right)+c_{\mathbf{b}}$ only translates that position in the normal direction; the coordinate transformations do not move the point. Consequently, when $c_{\mathbf{b}}>0$, the height of $\mathbf{b}_{c}^{\phi \lambda h}$ is too low and vice versa. 


\section{Base HI is reported too high}

If there is a constant positive HI blunder at the base, then we can modify equation (4) by adding a blunder constant to the true $\mathrm{HI}$ at $\mathbf{a}$ and $\mathbf{b}$ resulting in

$$
\begin{aligned}
& \mathbf{b}_{h}^{\phi \lambda h}= \\
& \chi^{x y z \rightarrow \phi \lambda h}\left(\chi_{\mathbf{b}}^{\text {neu } \rightarrow x y z}\left[\chi_{\mathbf{b}}^{x y z \rightarrow \text { neu }}\left\{\chi_{\mathbf{a}}^{\text {neu } \rightarrow x y z}\left(\left(0,0, H I_{\mathbf{a}}\right)+c_{\mathbf{a}}^{+}\right)+\mathbf{v}\right\}-\left(0,0, H I_{\mathbf{b}}+c_{\mathbf{b}}\right)\right]\right)
\end{aligned}
$$

where $c_{\mathbf{a}}^{+}$and $c_{\mathbf{b}}$ are real constants, and $\mathbf{b}_{h}^{\phi \lambda h}$ denotes the incorrectly computed position of $\mathbf{b}$; the subscript $h$ indicates that the base $\mathrm{HI}$ is too high. Therefore, $c_{\mathbf{a}}^{+}>0$. As before, when $c_{\mathbf{b}}>0$, then the HI reported at the rover is too high and vice versa. See Figure 4.
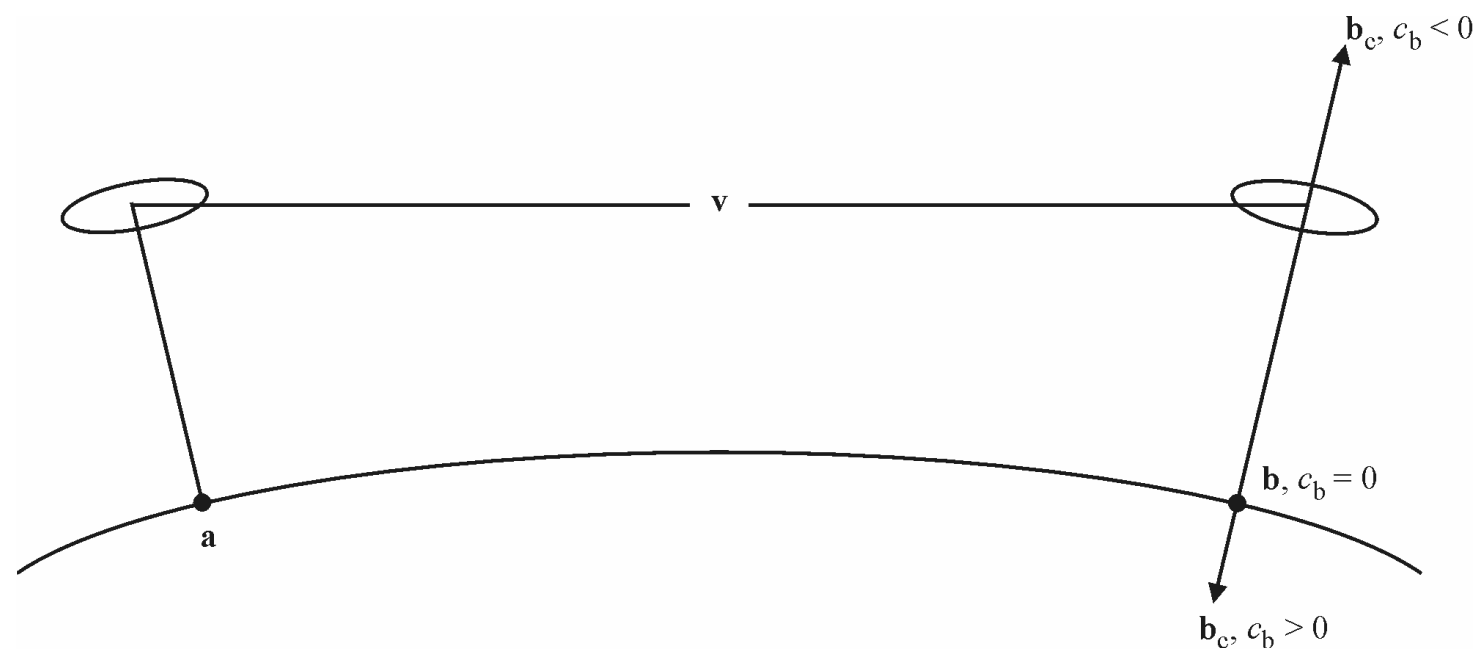

Fig. 3: A blunder only at the rover results in shifting b's position in a purely vertical fashion.

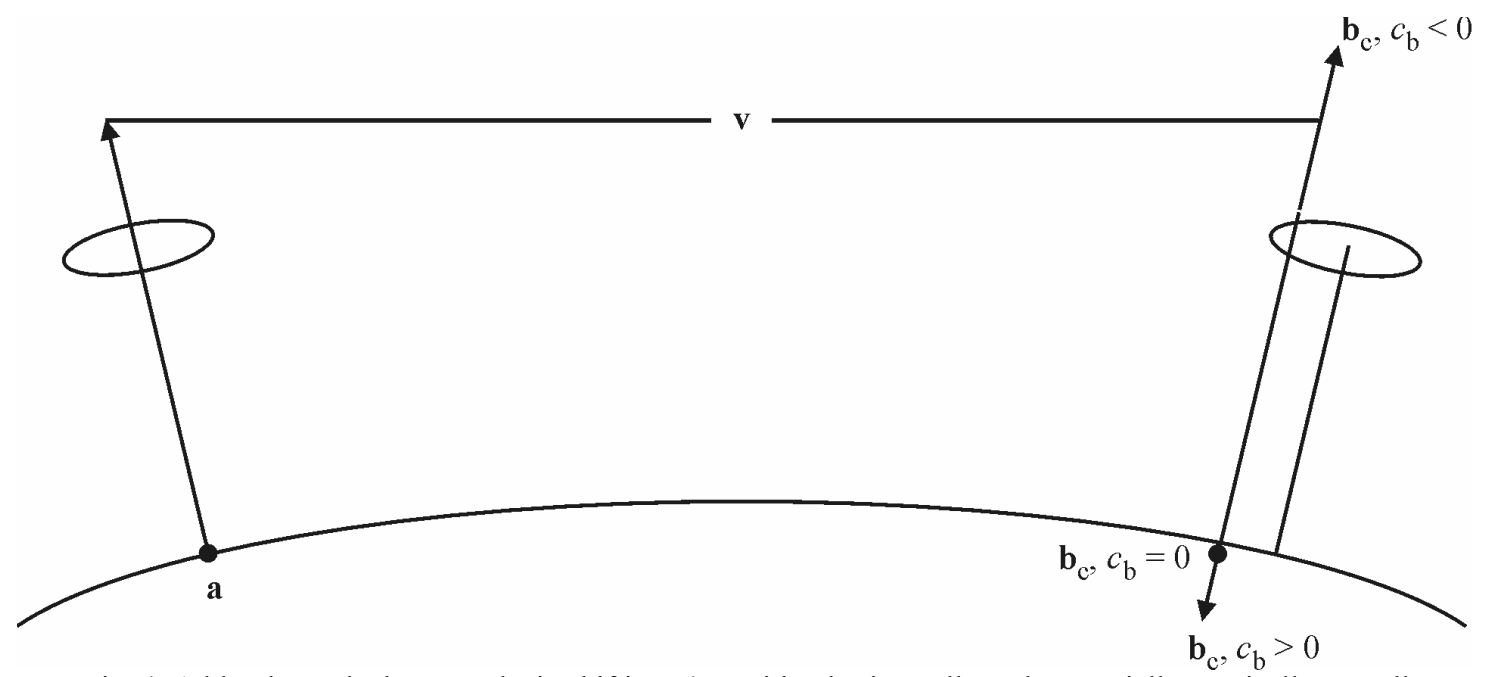

Fig. 4: A blunder at the base results in shifting b's position horizontally and, potentially, vertically as well.

Examination of equation (4b) reveals that $\mathbf{b}_{h}^{\phi \lambda h}$ differs from $\mathbf{b}^{\phi \lambda h}$ in both the horizontal and normal directions; the head of $\mathbf{v}$ has been misplaced and is no longer at $\mathbf{b}_{\phi}^{\phi \lambda h}$. This is true because the two range poles are not parallel due to the curvature of the Earth. By exaggerating the HI of the base, v's tail has been positioned too far apart from its correct location resulting in a computed $\mathbf{b}_{\phi}^{\phi \lambda h}$ that is too close to $\mathbf{a}$. Thereafter, the effect of $c_{\mathbf{b}}$ is much as in the previous scenario. However, as $\mathbf{a}$ and $\mathbf{b}$ are placed further apart, the error becomes more horizontal until it is worst at a separation of $90^{\circ}$. 
Figure 5 shows the resulting position error as determined in b's local $N E U$ coordinate system.
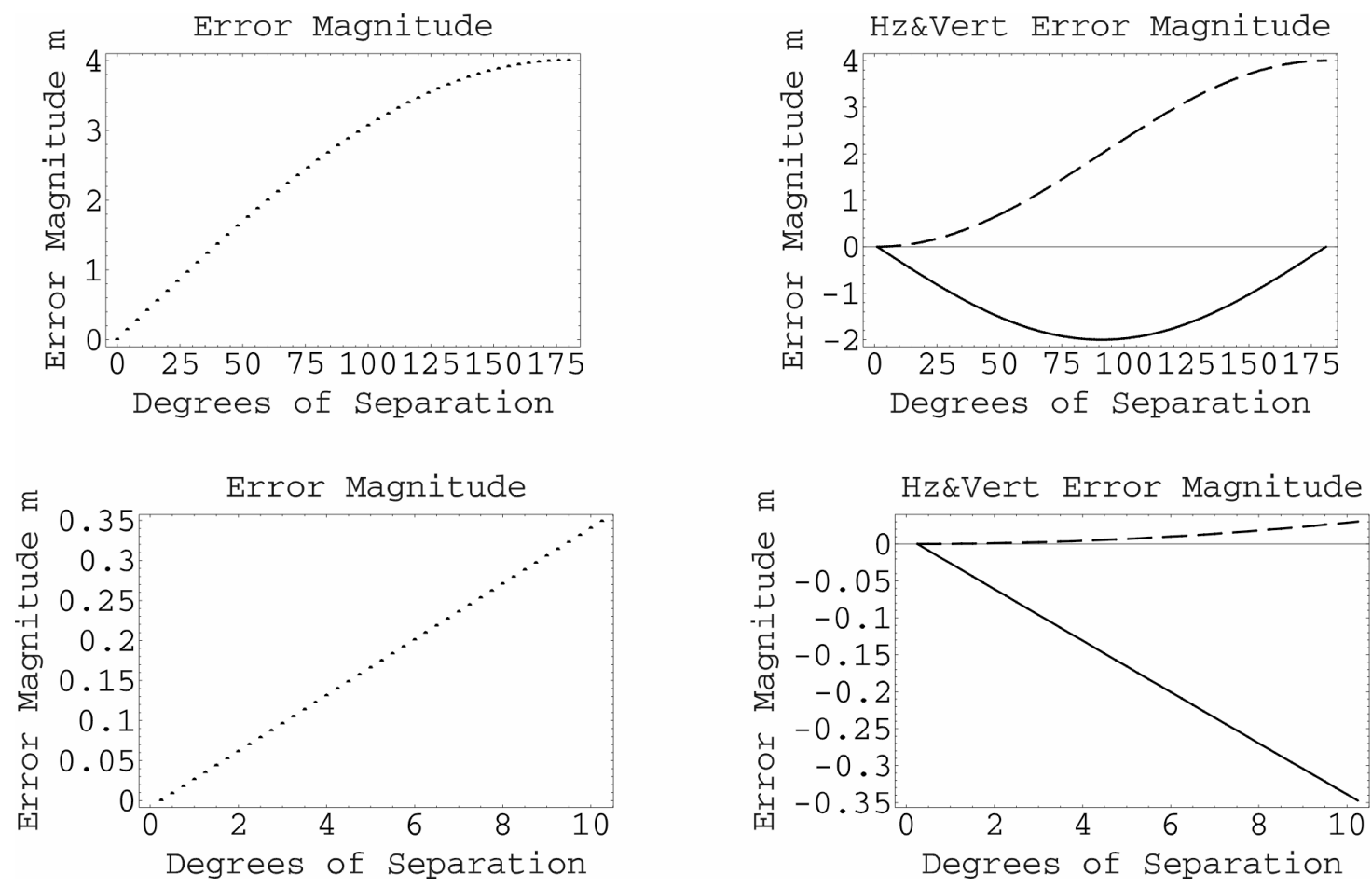

Fig. 5: Horizontal and vertical position displacements caused by a $2 \mathrm{~m} \mathrm{HI}$ blunder at both receivers. Solid lines are horizontal displacements. Dashed lines are vertical displacements. Dotted lines are 3D displacement magnitudes.

The two upper panels show the errors across the entire $180^{\circ}$ separation range. The upper left panel shows the magnitude of the three-dimensional error and the upper right panel separates the error into horizontal (solid line) and vertical (dashed line) components. Notice that the vertical error reaches a maximum with a $180^{\circ}$ separation. At $180^{\circ}$ of separation, the receivers are at antipodal nodes and all of the error is vertical, the magnitude of which is the sum of the two HI values, which were taken to be 2 metres each. The horizontal error achieves a maximum at $90^{\circ}$ from the base receiver, although this situation does not have the greatest overall error. The two lower panels show the errors across a $10^{\circ}$ separation range, which is perhaps more realistic for most surveyors. The three-dimensional error, shown on the left, is essentially linear. The lower right panel shows that, for these distances, almost all the error is horizontal (solid line). We note that separations larger than $90^{\circ}$ are typically not physically realizable for ground surveyors due to the lack of mutual satellite intervisibility. Nevertheless, we include the unrealizable separations for didactic purposes.

\section{Base HI is reported too low}

If there is a constant negative HI blunder at the base, then we can modify equation (4) by adding a blunder constant to the true $\mathrm{HI}$ at $\mathbf{a}$ and $\mathbf{b}$ resulting in

$$
\begin{aligned}
& \mathbf{b}_{l}^{\phi \lambda h}= \\
& \chi^{x y z \rightarrow \phi \lambda h}\left(\chi_{\mathbf{b}}^{\text {neu } \rightarrow x y z}\left[\chi_{\mathbf{b}}^{x y z \rightarrow n e u}\left\{\chi_{\mathbf{a}}^{\text {neu } \rightarrow x y z}\left(\left(0,0, H I_{\mathbf{a}}\right)+c_{\mathbf{a}}^{-}\right)+\mathbf{v}\right\}-\left(0,0, H I_{\mathbf{b}}+c_{\mathbf{b}}\right)\right]\right)
\end{aligned}
$$


where $c_{\mathbf{a}}^{-}$and $c_{\mathbf{b}}$ are real constants, and $\mathbf{b}_{l}^{\phi \lambda h}$ denotes the incorrectly computed position of $\mathbf{b}$; the subscript $l$ indicates that the base $\mathrm{HI}$ is too low. Therefore, $c_{\mathrm{a}}^{-}<0$. As before, when $c_{\mathbf{b}}>0$, then the $\mathrm{HI}$ reported at the rover is too high and vice versa. Examination of equation (4c) reveals that $\mathbf{b}_{l}^{\phi \lambda h}$ differs from $\mathbf{b}^{\phi \lambda h}$ by being too far apart. The situation is exactly the opposite as for equation (4b) due to the sign change in the blunder constant for $\mathbf{a}$, namely, $\mathbf{b}$ will always be computed to be farther from $\mathbf{a}$ than the truth.

\section{An example}

The three scenarios fully describe the nature of the problem but it is instructive to present an example of the problem's potential impact on practicing surveyors. Therefore, we consider the positioning mistakes that could result from a fairly gross HI blunder in the context of airborne GPS for topographic mapping with a scanning laser altimeter (LIDAR). For LIDAR, GPS positions are the basis by which LIDAR measurements are positioned on the Earth, so an understanding of how HI blunders effects such positions is essential.

Suppose we have an aircraft outfit with a survey-grade GPS receiver and a similar base station located at a known location. Let the region to be mapped be a square $2^{\circ}$ on a side with the base station exactly in the centre at $\left(42^{\circ} \mathrm{N}, 72^{\circ} \mathrm{W}\right)$. Now suppose that the HI of the base station is input as an orthometric height instead of an ellipsoid height, effectively causing an HI blunder of, say, 30 metres at the base. The resulting offsets are shown in Figure 6 as vectors whose tails are on the true locations and whose heads are at the offset locations. The longest vector in the Figure has a magnitude of 0.743 metres and all the other vectors are scaled linearly accordingly. Careful examination of Figure 6 will show that the displacement vectors do not emanate radially from the centre. Instead, they show a slight curving pattern. This curvature is caused by surface normal vectors along a normal section being skewed to one another. Also, the displacement vectors are symmetric east-to-west but not north-to-south. This is due to the convergence of the meridians.

Although the figure shows only horizontal displacements, LIDAR positions computed under such circumstances will be shifted by the three-dimensional error vector. Therefore, HI blunder errors are introduced both the horizontal and vertical coordinates of the LIDAR positions.

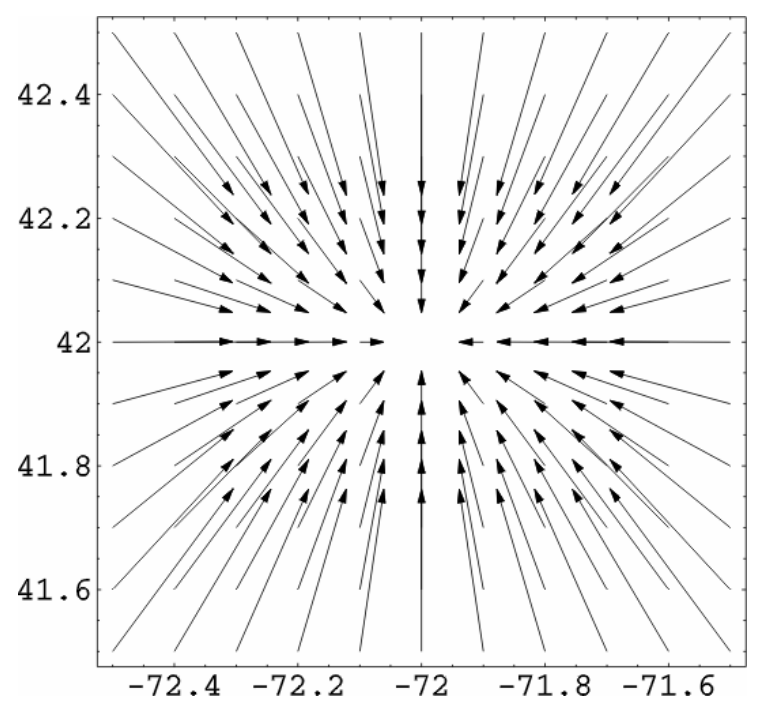

Fig. 6: Horizontal position displacements caused by a $30 \mathrm{~m} \mathrm{HI}$ blunder at the centre of the region. The longest vector corresponds to a horizontal displacement of $0.743 \mathrm{~m}$. Shorter vectors are scaled linearly. 


\section{EXPERIMENTAL VALIDATION}

We conducted a set of experiments to validate our theory. The data used in these experiments were taken from ten NGS CORS (National Geodetic Survey Continuously Operating Reference Stations) sites across the United States. For this study, the CORS station at Pennsylvania State University (PSU1: $40^{\circ} 48^{\prime} 24.81098^{\prime \prime} \mathrm{N}$,

$77^{\circ} 50^{\prime} 59.26862^{\prime \prime} \mathrm{W}, 312.54 \mathrm{~m}$ ) was chosen to be the base station. The rover stations were chosen so as to create a variety of baselines from hundreds to over $3000 \mathrm{~km}$ in length. The stations and their published NAD 83 (2002) coordinates are listed in the appendix. To remove any possible seasonal effects, data were taken from three different times of the year. We chose to use 12-hour duration occupations to ensure that there was enough data to obtain reliable results for the long baselines [2]. Therefore, each station contributed data from three, 12-hour occupations, therefore, each station's final coordinates were computed from three independent vectors.

The data were processed using Pinnacle, a proprietary GPS measurement processing program distributed by Topcon Positioning Systems. For this study, only the 9 vectors between PSU1 and the remaining stations where used, and PSU1 was held fixed to its published NGS coordinates. Because the theory of this study depends on the direction of the vector, we took pains to ensure that Pinnacle processed all the vectors starting at PSU1. All vectors were processed using precise ephemerides obtained from the U.S. National Geodetic Survey but with no other treatments. We did not process with tropospheric modeling due to a lack of meteorology data and Pinnacle does not support other treatments, such as ocean tide load [9] .

To validate our data and processing, we began by including all stations in a single network holding PSU1, HOPB and KWY1 as fixed control. Position errors, meaning the distance from our computed coordinates and those published by the NGS, are shown in Table 1. This network reproduced the NGS published horizontal coordinates at a $95 \%$ confidence level but failed to do so for some vertical coordinates, in one case by $37 \mathrm{~cm}$. After a careful investigation, we concluded that the discrepancy was due primarily to the different networks used to derive these heights. In specific, the NGS uses a relatively dense network of CORS stations whereas we used only three stations that are quite far apart. By adding additional control stations we were able to reduce the vertical errors. However, in the test cases that follow, we process vectors individually, not in a network, holding only PSU1 as fixed. Processing the vectors individually is necessary to eliminate the effect of the network least-squares adjustment Pinnacle applies, which distributes the errors throughout the network and confounds our purpose. Therefore, rather than give coordinates for a heavily constrained network, we felt these coordinates were more representative of how they would be used below.

Table 1 Horizontal and vertical discrepancies between published NGS coordinates and computed coordinates

\begin{tabular}{ccc}
\hline Point Name & Horz Dist $(\mathrm{m})$ & Vert Dist $(\mathrm{m})$ \\
\hline AZCN & 0.116 & -0.142 \\
\hline CCV3 & 0.057 & -0.087 \\
\hline COSO & 0.045 & 0.369 \\
\hline FBYN & 0.060 & -0.233 \\
\hline FERN & 0.169 & -0.097 \\
\hline PIT1 & 0.042 & -0.033 \\
\hline UVFM & 0.026 & -0.092 \\
\hline
\end{tabular}


Three distinct test cases where chosen to verify our theory. The first test case was to test the error at the base: the base station being located too high and the rover at the correct height. As discussed above it was expected that this would result in error both horizontally and vertically. A horizontal error is a displacement from the NGS published coordinates in a latitudinal direction, a longitudinal direction, or both. A vertical error is the difference in e ellipsoidal height from that published by the NGS. The second test case was to test the error at the rover by setting the base station correct and the rover too low, where a purely vertical mistake was expected. The final test case was simulating an error at both the base and rover instruments. In this case we chose to set the base too low and the rover too high, expecting that the error would be both horizontal and vertical and the overall vertical error would exceed the error introduced on either end. For all test cases an error with a magnitude of $30 \mathrm{~m}$ was used.

The results from these three test cases are presented in Figures 7 and 8 below. Figure 7 shows the horizontal distance between the NGS published coordinates and the coordinates produced in Pinnacle for the three test cases. As expected, the error increases linearly with baseline length. The observed horizontal errors for the second and third case match their theoretical predictions so closely that the graph of the prediction is indistinguishable from the experimental results. However, the horizontal error in the fourth test case should be essentially the same as the second; Pinnacle's computed coordinates were further away than we expected. We cannot explain the difference and we received no feedback from TOPCON technical support. An error at the base and not the rover produces a mix of horizontal and vertical mistakes, as expected. A mistake only at the rover reproduces the horizontal coordinates at the same $20 \mathrm{~cm}$ level found in the no error case, and an average $30 \mathrm{~m}$ error in the vertical direction. The case of error at both the base and the rover produced the greatest vertical error by placing the point around $60 \mathrm{~m}$ below where it should be.

\section{DISCUSSION AND CONCLUSIONS}

Perhaps the most surprising result of this study is that there are three different error regimes depending on whether the HI blunder is at both receivers, just at the base station, or just at the rover. Having such a complicated situation only stresses the importance of inputting correct HI values with GPS surveying because it becomes practically impossible to visually detect such errors after the network has been adjusted. If the HI blunders are random, such as might be the case if operators input a constant HI value and the true values were distributed around that value, then the resulting errors would be a mixture of displacements towards and away from the base.

We have shown that HI blunders can manifest as pure vertical errors, pure horizontal errors, and mixtures of everything in-between. The flight example shows how distorted a network can become due to these effects. Granted, a 30 metre blunder is a major mistake but confusing orthometric and ellipsoid heights is a realistic possibility. The resulting "contraction" of the adjusted network would be a significant problem and it might be very difficult to determine the cause.

In summary, HI blunders in GPS measurements cause position errors. These errors can be pure vertical, pure horizontal, or a mixture of both. There are different error regimes depending on whether both the base and the rover have HI blunders, if just the base has an HI blunder, or just the rover has an HI blunder. The resulting errors are on the order of $30 \mathrm{~cm}$ for receiver separations of $1000 \mathrm{~km}$ for an HI blunder of $2 \mathrm{~m}$. Given the complicated nature of the errors we believe it would be difficult, if not impossible, to detect such errors by visual inspection. This serves to underline the necessity to 
enter GPS HI's correctly and perhaps warrants double-checking them to ensure such errors are not introduced into the results.

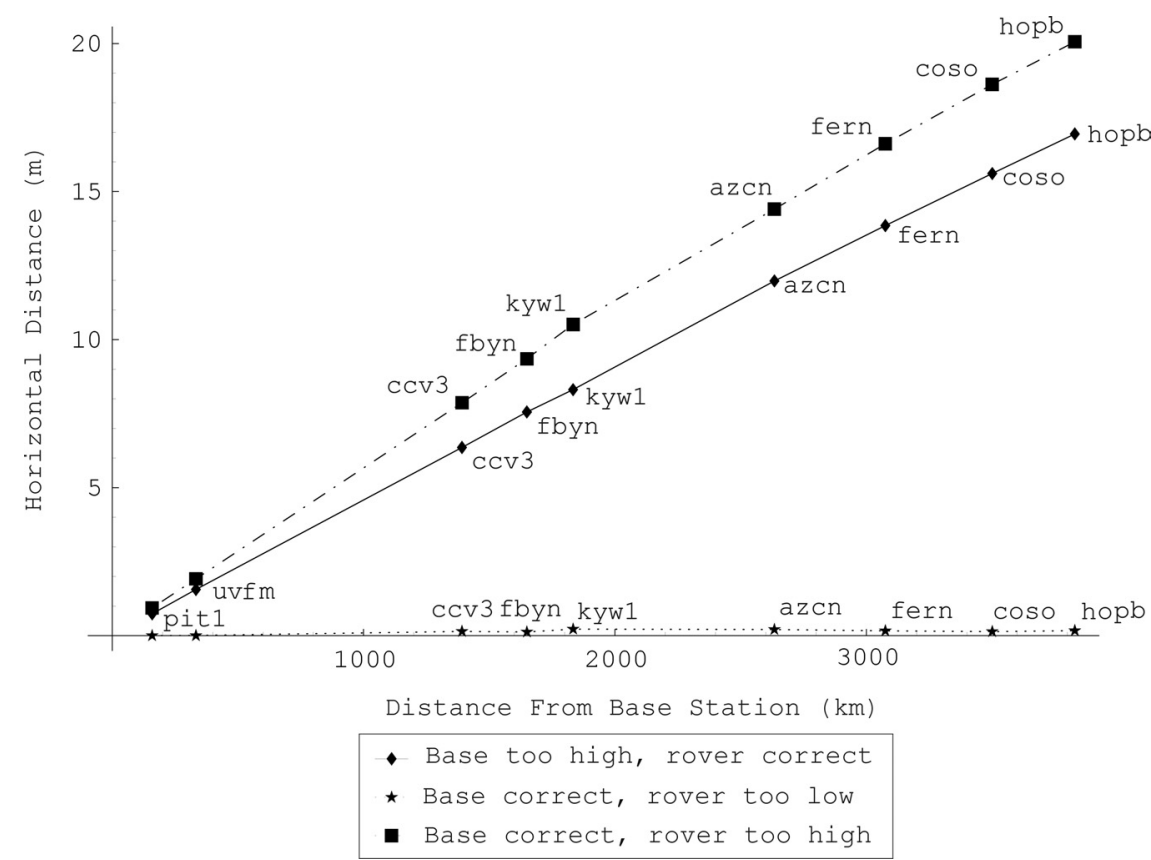

Fig. 7: Horizontal error for HI blunder test cases: the vertical axis represents the inverse horizontal difference between the coordinates found with a blunder introduced and the NGS published coordinates.

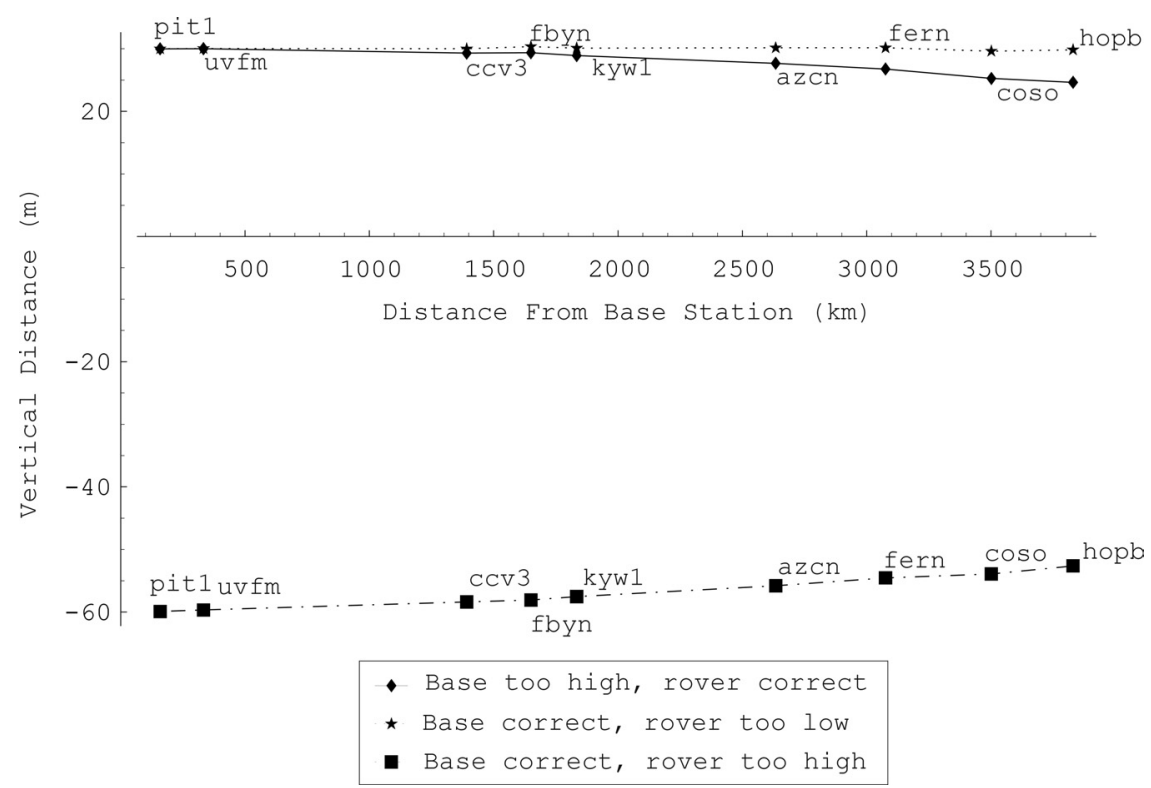

Fig. 8: Vertical error for HI blunder test cases: the vertical axis represents the ellipsoid height difference between the coordinates found with a blunder introduced and the NGS published coordinates

\section{References}

1. Bowring, B. R. Transformation from spatial to geographical coordinates. Survey Review. 1976; XXIII(181):pp. 323-327.

2. Eckl, M. C., Snay, R. A., Soler, T., Cline, M. W., and Mader, G. L. Accuracy of GPSderived relative positions as a function of interstation distance and observing-session duration. Journal of Geodesy. 2002; 75(12):pp.633-640.

3. Ewing, Clair E. and Mitchell, Michael M. Introduction to Geodesy. New York: 
American Elsevier Publishing Company, Inc.; 1970. 304 pp. .

4. Heiskanen, Weikko A. and Moritz, H. Physical Geodesy. New York: W. H.

Freeman \& Co., 1967. 364 pp.

5. Hofmann-Wellenhof, B., Lichtenegger, H., and Collins, J. GPS Theory and Practice. 4th ed. New York: SpringerWien NewYork; 1997. 389pp

6. Jones, G. C. New solutions for the geodetic coordinate transformation. Journal of Geodesy. 2002, 76(8):pp.437-446.

7. Leick, Alfred. GPS Satellite Surveying. 2nd ed. New York: John Wiley \& Sons, 1995. 560pp.

8. Meyer, Thomas H. Grid, Ground, and Globe: Distances in the GPS Era. Surveying and Land Information Science. 2002 Sep; 62(3):pp.179-202.

9. Seeber, Günter. Satellite Geodesy. 2nd ed. New York: Walter de Gruyter; 2003. 589pp.

10. Strang, Gilbert and Kai, Borre . Linear algebra, geodesy, and GPS. Wellesley, MA: Wellesley-Cambridge Press; 1997.

11. ---. personal communication. 2003.

12. Torge, Wolfgang. Geodesy. 2nd ed. New York: Walter du Gruyter; 1997. 264pp .

13. Wolf, Paul R. and Dewitt, Bon A. Elements of Photogrammetry with Applications in GIS. 3rd ed. Boston: McGraw Hill; 2000. 608pp .

\section{APPENDIX}

\begin{tabular}{|c|c|c|c|c|c|c|}
\hline & $\begin{array}{l}\text { Site } \\
\text { Name }\end{array}$ & Location & BLH Coords & XYZ Coords & $\begin{array}{c}\text { Baseline } \\
\text { Length }(\mathrm{km}) \\
\text { (Geodesic } \\
\text { distance } \\
\text { from PSU1 } \\
\text { to station) }\end{array}$ & $\begin{array}{c}\text { PID } \\
\text { (L1 } \\
\text { Phase } \\
\text { Centre) }\end{array}$ \\
\hline 1 & $\mathrm{AZCN}$ & $\begin{array}{l}\text { Aztec, New } \\
\text { Mexico }\end{array}$ & $\begin{array}{c}365023.23532 \mathrm{~N} \\
1075439.42216 \mathrm{~W} \\
1863.93 \mathrm{~m}\end{array}$ & $\begin{array}{l}X=-1572187.511 \\
Y=-4864407.313 \\
Z=3804296.249\end{array}$ & 2634.130 & AI0266 \\
\hline 2 & CCV3 & $\begin{array}{l}\text { Cape } \\
\text { Canaveral, } \\
\text { Florida }\end{array}$ & $\begin{array}{c}282736.79951 \mathrm{~N} \\
0803242.81940 \mathrm{~W} \\
-22.75 \mathrm{~m}\end{array}$ & $\begin{array}{l}X=921807.819 \\
Y=-5535344.708 \\
Z=3021430.002\end{array}$ & 1391.601 & AJ7943 \\
\hline 3 & $\mathrm{COSO}$ & $\begin{array}{l}\text { China Lake, } \\
\text { California }\end{array}$ & $\begin{array}{c}355856.41823 \mathrm{~N} \\
1174831.95366 \mathrm{~W} \\
1456.22 \mathrm{~m}\end{array}$ & $\begin{array}{l}X=-2411147.717 \\
Y=-4571431.854 \\
Z=3727461.640\end{array}$ & 3500.930 & AJ7939 \\
\hline 4 & FBYN & $\begin{array}{l}\text { Fairbury, } \\
\text { Nebraska }\end{array}$ & $\begin{array}{c}400436.71351 \mathrm{~N} \\
0971846.12084 \mathrm{~W} \\
406.42 \mathrm{~m}\end{array}$ & $\begin{array}{l}X=-622115.835 \\
Y=-4847773.066 \\
Z=4084781.523\end{array}$ & 1649.854 & AH9961 \\
\hline 5 & FERN & $\begin{array}{c}\text { Ferno Mesa, } \\
\text { Arizona }\end{array}$ & $\begin{array}{c}352030.72270 \mathrm{~N} \\
1122717.00717 \mathrm{~W} \\
1768.96 \mathrm{~m}\end{array}$ & $\begin{array}{l}X=-1989985.085 \\
Y=-4815007.272 \\
Z=3669893.915\end{array}$ & 3075.552 & AI8821 \\
\hline 6 & НOPB & $\begin{array}{l}\text { Hopland Bard, } \\
\text { California }\end{array}$ & $\begin{array}{c}385942.64677 \mathrm{~N} \\
1230428.96261 \mathrm{~W} \\
353.87 \mathrm{~m}\end{array}$ & $\begin{array}{l}X=-2708981.271 \\
Y=-4159580.753 \\
Z=3992123.807\end{array}$ & 3829.477 & AJ7940 \\
\hline 7 & KYW1 & $\begin{array}{l}\text { Key West, } \\
\text { Florida }\end{array}$ & $\begin{array}{c}243456.16453 \mathrm{~N} \\
0813910.90473 \mathrm{~W} \\
-12.06 \mathrm{~m}\end{array}$ & $\begin{array}{l}X=842465.076 \\
Y=-5741930.718 \\
Z=2637061.772\end{array}$ & 1833.844 & AJ7905 \\
\hline 8 & PIT1 & $\begin{array}{l}\text { Pittsburgh, } \\
\text { Pennsylvania }\end{array}$ & $\begin{array}{c}403303.73277 \mathrm{~N} \\
0794150.02303 \mathrm{~W} \\
354.28 \mathrm{~m}\end{array}$ & $\begin{array}{l}X=868033.524 \\
Y=-4775167.006 \\
Z=4124898.083\end{array}$ & 158.746 & AC7121 \\
\hline 9 & PSU1 & $\begin{array}{l}\text { State College, } \\
\text { Pennsylvania }\end{array}$ & $\begin{array}{c}404824.81098 \mathrm{~N} \\
0775059.26862 \mathrm{~W} \\
312.54 \mathrm{~m}\end{array}$ & $\begin{array}{l}X=1017620.251 \\
Y=-4726564.511 \\
Z=4146418.990\end{array}$ & ------------- & AE4425 \\
\hline 11 & UVFM & $\begin{array}{l}\text { University of } \\
\text { Virginia, Fan } \\
\text { Mountain, } \\
\text { Virginia }\end{array}$ & $\begin{array}{c}375243.46540 \mathrm{~N} \\
0784137.24953 \mathrm{~W} \\
514.38 \mathrm{~m}\end{array}$ & $\begin{array}{l}X=988331.390 \\
Y=-4943277.813 \\
Z=3895144.999\end{array}$ & 333.125 & AJ4871 \\
\hline
\end{tabular}

